\title{
BMJ Open Sex-specific effects of nutritional supplements in infants born early or small: protocol for an individual participant data meta-analysis (ESSENCE IPD-MA)
}

Luling Lin (D) , Caroline Crowther, Greg Gamble, Frank Bloomfield, Jane E Harding, The ESSENCE IPD-MA Group

To cite: Lin L, Crowther C, Gamble G, et al. Sexspecific effects of nutritional supplements in infants born early or small: protocol for an individual participant data meta-analysis (ESSENCE IPD-MA). BMJ Open 2020;10:e033438. doi:10.1136/ bmjopen-2019-033438

- Prepublication history and additional material for this paper are available online. To view these files, please visit the journal online (http://dx.doi org/10.1136/bmjopen-2019033438).

Received 05 August 2019 Revised 26 November 2019 Accepted 16 December 2019

D Check for updates

(c) Author(s) (or their employer(s)) 2020. Re-use permitted under CC BY-NC. No commercial re-use. See rights and permissions. Published by BMJ.

Liggins Institute, The University of Auckland, Auckland, New Zealand

Correspondence to Professor Jane E Harding; j.harding@auckland.ac.nz

\section{ABSTRACT}

Introduction Preterm and small for gestational age (SGA) infants are at increased risk of poor growth, disability and delayed development. While growing up they are also at increased risk of obesity, diabetes and later heart disease. The risk of such adverse outcomes may be altered by how preterm and SGA infants are fed after birth. Faltering postnatal growth is common due to failure to achieve recommended high energy and protein intakes, and thus preterm and SGA infants are often provided with supplemental nutrition soon after birth. Enhanced nutrition has been associated with improved early growth and better cognitive development. However, limited evidence suggests that faster growth may increase the risk for later adiposity, metabolic and cardiovascular disease, and that such risks may differ between girls and boys.

Methods and analysis We will search Ovid MEDLINE, Embase, Cochrane CENTRAL, Cochrane Database of Systematic Reviews, controlled-trials.com, ClinicalTrials. gov and anzctr.org.au for randomised trials that studied the effects of macronutrient supplements for preterm and SGA infants on (i) developmental and metabolic and (ii) growth outcomes after hospital discharge. The outcomes will be (i) cognitive impairment and metabolic risk (coprimary) and (ii) body mass index. Individual participant data (IPD) from all available trials will be included using an intention-to-treat approach. A one-stage procedure for IPD meta-analysis (MA) will be used, accounting for clustering of participants within studies. Exploratory subgroup analyses will further investigate sources of heterogeneity, including sex and size of infants, different timing, duration and type of supplements.

Ethics and dissemination This IPD-MA is approved by the University of Auckland Human Participants Ethics Committee (reference number: 019874). Individual studies have approval from relevant local ethics committees. Results will be disseminated in a peer-reviewed journal and presented at international conferences.

PROSPERO registration number CRD42017072683

\section{INTRODUCTION}

Infants born preterm or small for gestational age (SGA) are at increased risk of

\section{Strengths and limitations of this study}

- This individual participant data meta-analysis (IPDMA) will synthesise data from multiple randomised clinical trials using uniformly defined relevant outcomes for all available trials.

- Subgroup analyses may allow detection of potential sex differences that could not be achieved through aggregate analyses.

- This IPD-MA will explore the interactions between treatment and participant-level characteristics.

- A potential limitation is that the analyses planned will depend on obtaining the relevant individual participant data.

- There may be considerable heterogeneity as interventions are likely to be very varied and the trials performed over several decades.

poor growth, disability and delayed development. ${ }^{1-3}$ As adults, they are at increased risk of obesity, diabetes and later heart disease. ${ }^{4}$ How infants born small are fed after birth may alter the risk of these adverse outcomes. Providing preterm and SGA infants with enhanced nutrition soon after birth is associated with improved early growth and better cognitive development, ${ }^{5-7}$ but observational data suggest that early faster growth may increase the risk for later adiposity, metabolic and cardiovascular disease. ${ }^{8}$

It has been recognised for centuries that girls and boys grow differently, experience different metabolic and endocrine milieux and have different cognitive and health outcomes. Little attention has been paid to improving outcomes following preterm birth by treating girls and boys differently, although it is well recognised that preterm boys compared with girls have higher mortality and morbidity, ${ }^{9}$ and are more 
likely to have adverse developmental and educational outcomes. ${ }^{10}$ There is substantial evidence that perinatal insults can result in different adult phenotypes in males and females. ${ }^{11}$ For example, animal studies across many different species after a wide variety of prenatal insults show that males are more likely than females to exhibit adverse effects such as impaired renal function, hypertension, insulin resistance, altered hypothalamic-pituitaryadrenal axis function and altered growth in later life. ${ }^{12}$ The reasons for this sex difference in vulnerability to early environmental perturbations are not well understood, but may include faster growth and hence greater substrate demands in males, altered tempo of maturation, different exposure to sex steroids and sex-specific epigenetic mechanisms. ${ }^{12}$

There is limited evidence from human studies that these effects may also be important in babies. Preterm boys have been reported to have higher protein and calorie requirements to maintain growth than girls. ${ }^{13}$ Preterm boys also were more vulnerable than girls to the adverse effects of delayed early nutrition, ${ }^{14}$ whereas enhanced nutrition improved cognitive outcomes for preterm boys, but had no effect on girls. ${ }^{15}$ Unfortunately, most clinical studies have not reported findings separately by sex and are not adequately powered to do so. Further, because the majority of animal experiments are done in polytocous species, prenatal and postnatal sex effects cannot be separated in mixed-sex litters. There is little reliable evidence about how best to feed preterm babies to optimise both short-term and long-term health outcomes, and almost none about targeting nutrition by sex.

\section{Hypotheses}

The effects of early nutritional supplements on postdischarge development, markers of metabolic risk and growth are different in girls and boys.

\section{Need for individual participant data meta-analysis}

Systematic reviews using aggregate data meta-analyses are limited due to within-trial variation in gestational age of the infants at birth, comorbidities of the infant, starting time and duration of the intervention, macronutrient content of the intervention and control groups. Outcomes within aggregate meta-analyses such as cerebral palsy, motor dysfunction or hearing loss also include a range of severity of disability. Aggregate meta-analyses tend to vary in completeness and in the definitions used for the outcomes. Not all trials combine the same outcomes in composite outcomes or use the same measures of neurodevelopmental outcome. Few trials describe multiple subgroups, making meta-analysis of data almost impossible. Importantly, few trials to date have provided the sex of the infant as a subgroup variable.

One method to ameliorate some of the limitations of aggregate data meta-analysis is to combine the large volume of individual trial data available to perform an individual participant data (IPD) meta-analysis (MA). The estimates of treatment effects with an IPD-MA often differ from aggregate meta-analyses. ${ }^{16}$ IPD-MA allows the inclusion of additional unpublished data provided by the trialists, and allows consistent re-categorisation of definitions of outcomes and populations in order to answer the clinical questions of interest. Further, IPD-MA may allow more detailed meta-analysis of key outcomes, taking into account both subject-level and study-level sources of heterogeneity in treatment effects. Thus, IPD-MA offers the potential to help clarify the sex-specific effects of early macronutrient supplements provided to preterm and SGA infants.

\section{METHODS AND ANALYSIS}

This study will use an IPD-MA approach and follow the Preferred Reporting Items for Systematic Reviews and Meta-Analyses (PRISMA) guidelines. ${ }^{17}$

\section{Aims}

To assess, using IPD-MA, the effects of macronutrient supplements in nutrition of preterm and SGA infants on developmental, metabolic and growth outcomes after hospital discharge and in particular, whether these effects differ in girls and boys.

\section{Criteria for inclusion and exclusion}

We will include published and unpublished randomised and quasi-randomised trials without restrictions on date of publication or language. Trials that studied infants born preterm ( $<37$ weeks' gestation) or born small (birth weight $<2.5 \mathrm{~kg}$ or $<10^{\text {th }}$ centile for gestational age) and in which the intervention was intended to increase the intake of one or more macronutrients (protein, carbohydrate, fat, energy content or protein to energy ratio), with the primary aim of improving growth and development will be included.

Interventions can be enteral or parenteral or a combination, commence at any time during the hospitalisation or after discharge from hospital and must be provided for a minimum duration of 1 week. Trials that report on comparisons between unsupplemented nutrition and supplemented nutrition with parenteral supplements, human breast milk supplements, formula milk or other macronutrients will be eligible for inclusion, for example: (1) Parenteral formulation A versus parenteral formulation $\mathrm{B}$ with different macronutrient composition, (2) Human milk (mother's own or donor) versus supplemented human milk (mother's own or donor), (3) Human milk (mother's own or donor) versus formula milk (term or preterm), (4) Supplemented human milk (mother's own or donor) versus formula (term or preterm), (5) Supplemented human milk A (mother's own or donor) versus supplemented human milk B (mother's own or donor), (6) Formula A versus formula B with different macronutrient composition (including preterm vs term formula, brand A vs brand B). We will exclude trials that examine the timing of the introduction of nutrition (early vs delayed feeding), that compare macronutrients 
of different composition (eg, different types of lipids or proteins), studies whose outcomes focus on gastrointestinal development rather than growth and development and studies reporting on variations in composition of micronutrients (including sodium, potassium, calcium, phosphorus, vitamins, other minerals, amino acids, fatty acids).

Outcome data must be reported beyond term equivalent age ( $>37$ weeks' postmenstrual age) or following discharge from hospital after birth. Where the data are available, the outcomes will be categorised and evaluated in toddlers (less than 3 years), childhood (3 to 8 years), adolescence (9 to 18 years) and adulthood (more than 18 years).

We plan to report the findings as two reviews, one reporting developmental and metabolic outcomes, and the other reporting growth outcomes.

\section{Developmental and metabolic outcomes}

The co-primary outcomes will be (1) Cognitive impairment: below $-1 \mathrm{SD}$ on standard tests of development (toddlers) or cognition/intelligence quotient (later ages) and (2) Metabolic risk (see online supplementary appendix 1 for definitions): any of overweight/obese, increased waist circumference, increased fat mass or fat mass percentage, elevated plasma triglyceride concentrations, low high-density lipoprotein (HDL) concentrations, elevated low-density lipoprotein (LDL) concentrations, elevated fasting plasma glucose concentrations, insulin resistance, impaired glucose tolerance, diagnosis of type 2 diabetes, high blood pressure and impaired flowmediated vasodilatation.

The secondary outcomes will be (see online supplementary appendix 2 for definitions) (1) Composite of survival free of any disability (including death, cerebral palsy, motor development delay or impairment, cognition/ intelligence delay or impairment, language delay, visual impairment, hearing impairment); (2) Cognition/intelligence delay or impairment; (3) Cognition/intelligence scores; (4) Motor delay or impairment; (5) Motor scores; (6) Cerebral palsy (any); (7) Severity of cerebral palsy; (8) Visual impairment; (9) Hearing impairment; (10) School performance; (11) Measures of psychological wellbeing; (12) Metabolic outcomes: waist circumference, overweight/obese, type 2 diabetes, blood lipid concentrations (triglycerides, HDL, LDL, HDL:LDL), fasting blood glucose concentration, insulin concentration, insulin resistance, glucose tolerance, insulin-like growth factor-I concentration; (13) Cardiovascular risk outcomes: blood pressure (systolic blood pressure, diastolic blood pressure, mean arterial pressure), flow-mediated vasodilatation, measures of sympathetic and parasympathetic tone, cardiac size and structure; (14) Brain development: whole brain, white matter and grey matter volumes and volumes of individual brain regions, brain maturation measured using MRI (white matter tracts, measures of diffusivity, myelination, surface folding), functional brain imaging; (15) Health outcomes: allergies (eczema, asthma, hay fever), respiratory function, hospitalisation (duration), healthcare utilisation; (16) Nutrition: feeding tolerance; intake (milk, energy), appetite, breast feeding; (17) Death (neonatal or later death up to the time of follow-up and cause of death); (18) Quality of life; 19) General health and use of healthcare resources; (20) Adverse events; (21) Cost.

\section{Growth outcomes}

The primary outcome will be body mass index (BMI) in childhood (3 to 8 years).

The secondary outcomes will be (1) Growth assessments: weight (raw data and $\mathrm{z}$ scores), length/height (raw data and $\mathrm{z}$ scores), head circumference (raw data and z scores), Ponderal Index, BMI, body composition (fat mass, fat free mass, measured by bioimpedance or dual-energy X-ray absorptiometry or skinfold thickness or other method); (2) Bone development: bone mineral content, volumetric bone mineral density, bone fractures; (3) Nutrition: feeding tolerance; intake (protein, energy); appetite; breastfeeding and duration.

\section{Search strategy}

We will search Ovid MEDLINE, Embase, Cochrane CENTRAL and Cochrane Database of Systematic Review from inception to identify eligible trials. We will also search for registered trials in Current Controlled Trials (www.controlled-trials.com), ClinicalTrials.gov (www.clinicaltrials.gov) and Australian and New Zealand Clinical Trials Registry (www.anzctr.org.au) to identify eligible ongoing studies. The full search strategy and search terms are available as online supplementary material (online supplementary appendix 1). Experts in the field and trialists will be asked if they can identify other published or ongoing trials. Potentially eligible trials that are not yet completed will not be included in this IPD-MA, but will be noted for inclusion in future updates.

\section{Identify studies}

The eligibility of trials will be assessed by two researchers. Discrepancies will be resolved by discussion. If IPD are unavailable from any eligible trial it will be included in the IPD-MA using aggregate data for sensitivity analysis where possible.

\section{Contact authors}

Authors of eligible studies will be invited to join the ESSENCE IPD-MA Collaborative group. We will identify contact information from the published trials. An initial email will be sent to the main trial author (corresponding author) providing them with the summary IPD-MA protocol. Another investigator from the study will be contacted if initial emails fail to receive a response, followed by phone calls if needed.

\section{Quality assessment}

We will assess the quality of the eligible trials using the methods specified in the Cochrane Handbook for Systematic Reviews of Interventions: ${ }^{18}$ (1) random sequence 
generation (selection bias); (2) allocation concealment (selection bias); (3) blinding of participants, personnel and outcome assessment (performance and detection bias); (4) incomplete outcome data (attrition bias); (5) selective reporting (reporting bias); (6) other bias (checking for bias due to problems not covered by (1) to (5) above).

\section{Development of the database}

We will develop a set of prespecified and defined variables for IPD-MA at both the outcome, participant and trial level (online supplementary appendix 3). We will seek data on all randomised infants which will be coded for anonymity (date of birth, study centre); baseline data for narrative purposes (birth weight, gestational age at birth, plurality, sex); details of the intervention and comparator (date of randomisation, allocated intervention, type and composition of intervention and comparator, enteral or parenteral administration, age at start of intervention, weight at start of intervention, duration of intervention) and the outcomes listed above.

Trialists will provide de-identified data in any format which will be recoded as required, verified and checked for consistency with published data and stored on a secure, password protected file on the University of Auckland servers. Data will only be accessible by authorised personnel in the ESSENCE Data Management Group.

Methodological details of the individual trials will be cross-checked against published reports, trial registration (where available) and trial data collection forms. Where inconsistencies are identified, discussions will be held with individual trial groups to attempt to resolve these. Each trial final data set to be used in the IPD-MA will be returned to the trialists for verification. Trials will then be analysed individually using IPD-MA prespecified variables and outcomes and the results returned to the trialists for verification. The individual trial data sets will then be combined to form the ESSENCE-IPD data set and the IPD-MA undertaken.

\section{Data synthesis and statistical analysis}

A detailed statistical analysis plan will be prepared and agreed on by the ESSENCE-IPD team.

We will use a one-stage approach to the analysis of each outcome so that the IPD from all eligible trials are included in a single model. We will make an assessment of heterogeneity to decide if combining data from trials is appropriate or if heterogeneity, if significant, can be explained.

Binary outcomes will be analysed using log binomial regression models and data will be reported as risk ratio with 95\% CIs and associated two-sided $\mathrm{p}$ values. Continuous data will be analysed using linear regression models and data will be reported as mean differences with $95 \%$ CIs and associated two-sided $\mathrm{p}$ values.

A large number of outcomes are being investigated in this study. This increases the chance of observing false positive results. The overall probability of a type 1 error will be maintained at $5 \%$ for each review. For the review of developmental and metabolic outcomes, the $\mathrm{p}$ value will be split equally between the co-primary outcomes by testing each at $p=0.025$. For the review of growth outcomes, $p<0.05$ will denote statistical significance for the primary outcome. No further adjustment for multiplicity is planned for comparisons made in secondary and exploratory analyses.

We will explore the effects of the sex of the infants by presenting data separately for each sex as prespecified subgroups, and by testing a treatment by sex interaction term within the model.

Where data are missing, those infants will be removed from the analysis and, where possible, the reasons for missing data will be explored. It is not proposed to impute missing data since the assumption of 'missing at random' is unlikely to be met. Where there are large amounts of missing data or trials are unable to provide IPD we will conduct sensitivity analyses to explore the effect of removing such trials from the analysis.

Statistical analyses will be performed using SAS (v.9.4, SAS Institute, Cary, North Carolina, USA).

\section{Planned subgroup analysis}

Where data are available, we will conduct subgroup analyses to explore whether the effects of supplements differ between subgroups and test for interaction terms.

1. Sex of infant (boys vs girls);

2. Size of infant at birth ( $\leq 1 \mathrm{~kg} v \mathrm{vs}>1 \mathrm{~kg}$ at birth);

3. Size for gestation of the infant $\left(\leq 10^{\text {th }}\right.$ centile vs $>10^{\text {th }}$ centile);

4. Gestational age of infant at birth ( $\leq 28$ completed weeks vs 29 to 32 completed weeks vs 33 to 36 weeks).

5. Timing of supplement:

In-hospital nutrition: the intervention was commenced in-hospital or on average ended at 42 weeks' postmenstrual age or earlier;

Post-discharge nutrition: the intervention was commenced after discharge or on average started at or after 36 weeks' postmenstrual age;

Both in-hospital and post-discharge nutrition: the intervention was commenced in the hospital and continued post-discharge.

6. Type of supplement (protein vs carbohydrate vs fat vs multicomponent and their interactions).

7. Breast milk versus formula as primary milk feed.

8. Duration of supplement ( 1 to 2 weeks vs 3 to 6 weeks vs more than 7 weeks).

9. Different epochs (commenced up to the year of 2000 vs commenced in or after the year of 2001).

\section{Planned sensitivity analyses}

We will perform sensitivity analyses to assess whether the results are robust to the trial design by excluding trials assessed as high risk of bias.

Where trials are unable to contribute data to the IPD we will assess the robustness of the inclusion or exclusion of these trials by combining their aggregate data with the IPD. 


\section{Patient and public involvement}

It was not appropriate or possible to involve patients or the public in the design, or conduct, or reporting or dissemination of our research.

\section{DISCUSSION}

This will be the first IPD-MA to investigate the effects of early macronutrient supplements on preterm and SGA infants. IPD-MA has been described as the 'gold standard' of systematic review methodology as it allows for more powerful and flexible analysis of both subgroups and outcomes. ${ }^{19}$ This IPD-MA, using existing data from the individual trials, may reveal the sex-specific effects of macronutrient supplements on preterm and SGA infants, and will allow assessment of important interactions that cannot be tested in standard, aggregate data meta-analysis.

\section{ETHICS AND DISSEMINATION}

The shared data will be de-identified, the data files will be transferred by secure means and stored in a secure password protected area on an Auckland University server.

Final results will be presented to the ESSENCE IPD-MA collaborators prior to publication and public dissemination. Results of the study will be published in peer-reviewed journals and presented at national and international conferences.

Acknowledgements We would like to acknowledge Dr Julie Brown for help with developing the draft protocol and search strategies, Mariana Muelbert for her help with the communication with trialists from Brazil and Laura Galante for her help with the communication with trialists from Italy.

Collaborators The ESSENCE IPD-MA Group ESSENCE-IPD Project Team: This is the project Steering Group which is responsible for the day-to-day management of the IPD. This group has drafted the IPD protocol, will liaise with trialists and prepare the draft publications. J.E. Harding (chair of ESSENCE-IPD project); L Lin; C.A. Crowther; F.H. Bloomfield and G Gamble. ESSENCE-IPD Management Group: This group is convened by the Chair of the Project Team and comprises the IPD-MA statistician and data manager who will be responsible for the collection, checking, storage and analyses of data. J.E. Harding (chair of ESSENCE-IPD project); L Lin; C.A. Crowther and G Gamble. ESSENCE-IPD Trialist Group: This group includes investigators from all eligible trials who have agreed to share their data for the IPD.M Agosti (Division of Neonatology and Neonatal Intensive Care Unit, 'F. Del Ponte' Hospital, Varese, Italy); S.A. Atkinson (Department of Pediatrics, Faculty of Health Sciences, McMaster University, Hamilton, Ontario, Canada.); A Biasini (Donor Human Milk Bank Italian Association (AIBLUD), Milan, Italy); R.D.S Da Cunha (Hospital Universitário da Universidade Federal do Maranhão - Brasil); N.D. Embleton (Institute of Health and Society, Newcastle University, Newcastle upon Tyne, UK); M Faraz (Department of Pediatrics, Faculty of Health Sciences, McMaster University, Hamilton, Ontario, Canada.); M.S Fewtrell (Childhood Nutrition Research Centre, UCL Great Ormond Street Institute of Child Health, London, UK); F Lamy Filho (Departamento de Medicina, Universidade Federal do Maranhão (UFMA), São Luís, MA, Brazil); C. Fusch (Department of Pediatrics, Faculty of Health Sciences, McMaster University, Hamilton, Ontario, Canada and Department of Pediatrics, Nuremberg General Hospital, Paracelsus Medical University, 90471 Nuremberg, Germany); M.L. Gianni (Fondazione IRCCS Cà Granda Ospedale Maggiore Policlinico, via Commenda 12, 20122 Milan, Italy); H.G. Kanmaz (Department of Neonatology, NICU, Zekai Tahir Burak Education andResearch Hospital, Ankara, Turkey); W.WK. Koo (Department of Nutrition and Food Science, Wayne State University, Detroit, MI, USA); I Litmanovitz (Department of Neonatology, Meir Medical Center, Kfar Saba, Israel); A Lucas (MRC Childhood Nutrition Research Centre, Institute of Child Health, University College London, London, UK); C Morgan (Department of Neonatology, Liverpool Women's Hospital, Liverpool, UK); K Mukhopadhyay (Department of Pediatrics, Post Graduate
Institute of Medical Education and Research (PGIMER), Chandigarh, India.); E Neri (Department of Psychology, University of Bologna, Bologna, Italy); J Picaud (Division of Neonatology, Hôpital de la Croix-Rousse, Lyon, France); E.V Rafael (Departamento de Enfermagem da Universidade Federal do Maranhão, Brasil); P Roggero (Fondazione IRCCS Cà Granda Ospedale Maggiore Policlinico, via Commenda 12, 20122 Milan, Italy); A Singhal (Department of Nutrition, Institute of Child Health, London, UK); K Stroemmen (Department of Neonatal Intensive Care, Division of Pediatric and Adolescent Medicine, Rikshospitalet, Oslo University Hospital, Oslo, Norway); M.J. Tan (Department of Developmental Paediatrics, Alder Hey Children's NHS Foundation Trust, Liverpool, UK); F.M. Tandoi (Division of Neonatology and Neonatal Intensive Care Unit, "F. Del Ponte" Hospital, Varese, Italy); C.L. Wood (Institute of Genetic Medicine, Newcastle University, Newcastle, UK); G Zachariassen (H.C. Andersen Children's Hospital, Odense University Hospital and University of Southern Denmark, Odense, Denmark)

Contributors The Chair of the ESSENCE-IPD Project Team (J.E. Harding) wrote the first draft of the ESSENCE-IPD protocol. L Lin revised the subsequent versions of the ESSENCE-IPD protocol and prepared the initial draft of the manuscript. The ESSENCE-IPD Project Team and the ESSENCE-IPD Management Group participated in the protocol development and commented on all drafts of the manuscript. The ESSENCE-IPD Trialist Group participated in the development of the IPD protocol and have read and approved the final draft of the manuscript.

Funding The ESSENCE-IPD project is supported by the Health Research Council (HRC) of New Zealand (16/605). The analysis will be included as part of the doctoral thesis of Luling Lin, who is supported by Agnes Paykel PhD Scholarship. None of the funders are involved in any other aspect of the project, such as the design of the protocol and analysis plan, the collection and analysis of the data or the interpretation and publication of the study results.

Competing interests None declared.

Patient consent for publication Not required.

Ethics approval The ESSENCE-IPD project has been approved by University of Auckland Human Participants Ethics Committee. Participants in the individual trials have previously given informed consent to participate in their respective trials. The data for these projects are to be used for the purpose for which they were originally collected and are available through an agreement between all trialists of the collaborative group. These trialists remain custodians of their original trial data at all times. A data sharing agreement will be signed by a representative of the institution that owns the data and a representative of University of Auckland.

Provenance and peer review Not commissioned; externally peer reviewed.

Open access This is an open access article distributed in accordance with the Creative Commons Attribution Non Commercial (CC BY-NC 4.0) license, which permits others to distribute, remix, adapt, build upon this work non-commercially, and license their derivative works on different terms, provided the original work is properly cited, appropriate credit is given, any changes made indicated, and the use is non-commercial. See: http://creativecommons.org/licenses/by-nc/4.0/.

ORCID iD

Luling Lin http://orcid.org/0000-0002-8448-1504

\section{REFERENCES}

1 Embleton NE, Pang N, Cooke RJ. Postnatal malnutrition and growth retardation: an inevitable consequence of current recommendations in preterm infants? Pediatrics 2001;107:270-3.

2 Hay WW. Nutritional requirements of extremely low birthweight infants. Acta Paediatr Suppl 1994;402:94-9. Jr.

3 Cooke RWI. Conventional birth weight standards obscure fetal growth restriction in preterm infants. Arch Dis Child Fetal Neonatal Ed 2007;92:F189-92.

4 Luu TM, Katz SL, Leeson P, et al. Preterm birth: risk factor for earlyonset chronic diseases. Can Med Assoc J 2016;188:736-46.

5 Lucas A, Fewtrell MS, Morley R, et al. Randomized outcome trial of human milk fortification and developmental outcome in preterm infants. Am J Clin Nutr 1996;64:142-51.

6 Lucas A, Morley R, Cole TJ. Randomised trial of early diet in preterm babies and later intelligence quotient. BMJ 1998;317:1481-7.

7 Lucas A, Morley R, Cole TJ, et al. A randomised multicentre study of human milk versus formula and later development in preterm infants. Arch Dis Child Fetal Neonatal Ed 1994;70:F141-6.

8 Peacock JL, Marston L, Marlow N, et al. Neonatal and infant outcome in boys and girls born very prematurely. Pediatr Res 2012;71:305-10. 
9 Glass HC, Costarino AT, Stayer SA, et al. Outcomes for extremely premature infants. Anesthesia \& Analgesia 2015;120:1337-51.

10 Ong KK, Kennedy K, Castañeda-Gutiérrez E, et al. Postnatal growth in preterm infants and later health outcomes: a systematic review. Acta Paediatr 2015;104:974-86.

11 Perera F, Herbstman J. Prenatal environmental exposures, epigenetics, and disease. Reproductive Toxicology 2011;31:363-73.

12 Aiken CE, Ozanne SE. Sex differences in developmental programming models. Reproduction 2013;145:R1-13.

13 Alur P. Sex differences in nutrition, growth, and metabolism in preterm infants. Front. Pediatr. 2019;7.

14 Poindexter BB, Langer JC, Dusick AM, et al. Early provision of parenteral amino acids in extremely low birth weight infants: relation to growth and neurodevelopmental outcome. J Pediatr 2006;148:300-5.
15 Lin L, Amissah E, Gamble GD, et al. Impact of macronutrient supplements for children born preterm or small for gestational age on developmental and metabolic outcomes: a systematic review and meta-analysis. PLoS Med 2019;16:e1002952.

16 Smith CT, Marcucci M, Nolan SJ, et al. Individual participant data meta-analyses compared with meta-analyses based on aggregate data. Cochrane Database Syst Rev 2016;9.

17 Moher D, Liberati A, Tetzlaff J, et al. Preferred reporting items for systematic reviews and meta-analyses: the PRISMA statement. PLoS Med 2009;6:e1000097.

18 Higgins JPT, Green S, The Cochrane collaboration. Cochrane Handbook for systematic reviews of interventions version 5.1.0. Available: www.cochrane-handbook.org [Accessed 9 Apr 2019].

19 Tierney JF, Vale C, Riley R, et al. Individual participant data (IPD) meta-analyses of randomised controlled trials: guidance on their use. PLoS Med 2015;12:e1001855. 